\title{
Interactive comment on "Validity and limitations of simple reaction kinetics to calculate concentrations of organic compounds from ion counts in PTR-MS" by Rupert Holzinger et al.
}

\section{Anonymous Referee \#2}

Received and published: 6 March 2019

This paper reports measurements by unique calibration protocol on 10 different PTRMS instruments and comparison with a reaction kinetic model. The authors conclude that the kinetic model accurately predicts the sensitivities if no artefacts interfere and the instruments were operated at $\mathrm{E} / \mathrm{N}$ levels above $100 \mathrm{Td}$. However, I have a question about the kinetic model, in terms of how to think the reaction time for quadrupole-type drift tube (comment 1). Also, kinetics for the PTR3 instrument should be discussed more deeply (comments 2 and 3 ). My major concerns should be addressed before accepted for publication. 
the residence time for all the instruments except for "PTR3 HAR". I have a question here: Can this equation be applied to the case of the instruments with quadrupole-type drift tubes (e.g., TOFqi and Vocus)? Eq. 11 is originally for static lens ion optics in which ions move linearly, and therefore, the drift tube length can be used directly for the calculation of the time. In contrast, ions move spirally in the quadrupole-type drift tube. This means ion trajectories are quite long compared to the drift tube length. The authors should clarify how they consider this matter.

2. Page 9, line 12 etc.: The authors state that primary ions undergo approximately 1000 times more collisions with the analyte gas in the PTR3 instrument. Taking into account this matter and Figure $A 1, I$ think that the following proton transfer reaction between analyte gas $(\mathrm{R})$ and ion $(\mathrm{RH}+)$ should be considered, as well as de-protonation $(\mathrm{RH}++$ $\mathrm{H} 2 \mathrm{O} \rightarrow \mathrm{H} 3 \mathrm{O}++\mathrm{R}$ ). $\mathrm{R} 1 \mathrm{H}++\mathrm{R} 2 \rightarrow \mathrm{R} 2 \mathrm{H}++\mathrm{R} 1$ (if $\mathrm{PA}(\mathrm{R} 2)>\mathrm{PA}(\mathrm{R} 1)$, PA: proton affinity) The authors should discuss how the analyte sensitivities are affected by this reaction in case of the PTR3 instrument. Additionally, the density of water molecules in the drift tube should be discussed if de-protonation is considered.

3. Figure $A 1$ in page 27: The measured sensitivities for isoprene and alpha-pinene are quite low compared to the expected ones. Do these results mean that excess fragmentation occurs in the PTR3 instrument? In the case of the PTR3 instrument, is there a possibility that CID (collision-induced dissociation)-like fragmentation occus? It would be good if the authors could suggest any reasons for quite low sensitivities for isoprene and alpha-pinene in the PTR3.

Minor concerns: 4. Page 27, line 19: "t_react" should be defined here or in the main text.

Interactive comment on Atmos. Meas. Tech. Discuss., doi:10.5194/amt-2018-446, 2019. 